\title{
Utility Optimal Scheduling in Energy Cooperation Networks Powered by Renewable Energy
}

\author{
Congshi Hu, Xu Zhang, Sheng Zhou, Zhisheng Niu \\ Tsinghua National Laboratory for Information Science and Technology, \\ Dept. of Electronic Engineering, Tsinghua Univ., Beijing, 100084, P. R. China \\ \{hucs11, zhang-xu10\}@mails.tsinghua.edu.cn, \{sheng.zhou, niuzhs\}@tsinghua.edu.cn
}

\begin{abstract}
In this paper, we consider the problem of energy and data control in energy cooperation networks powered by renewable energy. In such networks, nodes can provide data transmission service, and at the same time they have power lines to transfer the harvested energy to others. We develop an online algorithm called Energy and Data Aware (EDA) algorithm using Lyapunov analysis, which makes data admission control and decides energy allocation for traffic transmission and energy transfer. In our EDA algorithm, the node only needs to keep track of its own energy storage status and does not require any knowledge of the energy harvesting process. We show that the proposed algorithm achieves a utility that is within $O(\varepsilon)$ of the optimal, for any $\varepsilon>0$, while ensuring that both the network data queue length and the capacity of energy storage devices are upper bounded by bounds of size $O(1 / \varepsilon)$.
\end{abstract}

\section{INTRODUCTION}

Energy harvesting has received more and more attentions in wireless communication systems because of its environmental friendship and convenient deployment. In such systems, transmitters can harvest energy from random energy resources, such as sun or wind. Recently, equipping Base Stations (BSs) with renewable energy has been proved technically feasible [1]. However, the harvested energy varies both in the time domain and the space domain [2], and the energy harvesting process is typically hard to predicate, which both lead to new challenges for resource management.

We consider an energy cooperation scenario which can help to solve the problem of imbalanced harvested energy in space domain. There are multiple nodes in the network and each node can transfer its harvested energy to some others. For every time slot, the controller decides how much data is admitted and how the energy is allocated between data transmission and energy transfer. The objective of the network is to maximize the traffic utility subject to the constraints that the network is stable, which means the queue length is always bounded, and satisfies the energy casuality. Here the energy casuality means that at every time the energy consumed cannot be more than the energy received.

In literature, there have been substantial research efforts to address the challenges with the arrival dynamics of renewable energy. For the single node scenario, existing papers mainly focus on the power allocation problem. For the non-causal

This work is sponsored in part by the National Basic Research Program of China (2012CB316001), the Nature Science Foundation of China (61201191, 60925002, 61021001), and Hitachi Ltd. information condition, some efforts have been done to find the structure of the optimal power allocation to maximize the throughput and the directional water-filling algorithm has been proposed [3]- [5]. And in our previous work [6], we propose the optimal spatial-temporal water-filling power allocation for the MIMO system. For networks with multiple nodes, assuming the harvested energy satisfies a leaky-bucket type property, Ref. [7] considers the optimal power management for sensor nodes. Using diffusion approximation, Refs. [8] [9] find the analytic expression of the energy outage probability in wireless mesh networks. Ref. [10] considers the problem of utility optimal scheduling in multi-hop networks. However, most of the existing papers focus on independent energy conditions, which means that nodes cannot share its energy with each other. Furthermore, much work requires sufficient statistical knowledge of the harvested energy, which is actually hard to obtain. Recently, there are some papers considering energy cooperation issues to use the harvested energy more efficiently, including cellular network planning [11] and power gird energy saving [12]. But still, how to run networks with energy cooperation is still an open issue.

For our energy allocation and data admission problem in the proposed energy cooperation scenario, we tackle it by using the Lyapunov optimization developed in [13] and [14]. Based on the Lyapunov function, we propose an Energy and Data Aware (EDA) algorithm to achieve the near-optimal utility in the energy cooperation networks. This algorithm is an online algorithm which makes decisions slot-by-slot without the knowledge of the energy harvesting process. We show that the EDA algorithm is able to achieve a utility within $O(\varepsilon)$ of the optimal for any $\varepsilon>0$ while the network data backlog and energy storage device capacity are both upper bounded by bounds of size $O(1 / \varepsilon)$.

This paper is organized as follows. Section II presents the system model. In section III, we analyze this problem by using the Lyapunov optimization and give the performance analysis of the proposed algorithm. Simulation results are given in Section IV. And finally, Section V concludes this work.

\section{SyStem MODEL}

We consider a time-slotted network. Each node in the network can harvest energy from random sources, such as sun or wind. We assume the harvested energy is used only for transmission, which means the processing energy is ignored. 
The network is modeled by a directed graph $\mathcal{G}=\{\mathcal{L}, \mathcal{N}\}$, where $\mathcal{N}=\{1,2, \ldots, N\}$ denotes the set of $N$ server nodes and $\mathcal{L}=\{[n, m], n, m \in \mathcal{N}\}$ denotes the set of energy transfer links in the network. Here an energy transfer link $[n, m]$ means the harvested energy can be transferred from node $n$ to node $m$.

We denote $\mathcal{N}_{n}^{(\text {out })}$ as the set of nodes $b$ with $[n, b] \in \mathcal{L}$ and $\mathcal{N}_{n}^{(i n)}$ as the set of nodes $a$ with $[a, n] \in \mathcal{L}$. We also define

$$
d_{\max } \triangleq \max _{n}\left(\left|\mathcal{N}_{n}^{(\text {in })}\right|,\left|\mathcal{N}_{n}^{(\text {out })}\right|\right),
$$

as the maximum in-out degree that any node can have.

\section{A. Data Queue Model}

In each node, there is a buffer to store the admitted data. Let $A_{n}(t)$ denote the admitted data in node $n$ (in bits) of time slot $t$ and let $\gamma_{n}(t)$ denote the channel gain that node $n$ is facing with, which takes values in some finite set $\left\{\gamma_{1}, \gamma_{2}, \ldots, \gamma_{M_{n}}\right\}$. For node $n$, the power allocated to transmit data is $0 \leq P_{n}(t) \leq P_{\max }$ for some $P_{\max }<\infty$. Given the channel state and power allocation, the transmission rate is given by $\mu_{n}(t)=\mu_{n}\left[P_{n}(t), \gamma_{n}(t)\right]$ and is assumed to be upper bounded by some linear function of the allocated power, i.e.,

$$
\mu_{n}\left[P_{n}(t), \gamma_{n}(t)\right] \leq \delta P_{n}(t) .
$$

For instance, the Shannon equation is one example following this assumption.

In this paper, we assume that there is no interference among nodes and that $0 \leq A_{n}(t) \leq A_{\max }, 0 \leq \mu_{n}(t) \leq \mu_{\max }$ for some $A_{\max }<\infty$ and $\mu_{\max }<\infty$. Then the queue length $Q_{n}(t)$ evolves as following

$$
Q_{n}(t+1)=\left[Q_{n}(t)-\mu_{n}(t)\right]^{+}+A_{n}(t),
$$

where $[x]^{+}=\max \{0, x\}$.

\section{B. Energy Queue Model}

Each node has an energy buffer to store the harvested energy and the energy arrived from other nodes. We assume the energy queue has infinite buffer size and later we will show that our policy makes the energy buffer finite later. As mentioned before, if there is an energy link $[n, m]$, then energy can be transferred from $n$ to $m$. The newly transferred energy can be used at the next time slot. We use $\hat{h}_{n}(t) \in\left\{\hat{h}_{1}, \hat{h}_{2}, \ldots, \hat{h}_{M_{h}}\right\}$ to denote the amount of harvestable energy and $h_{n}(t) \leq \hat{h}_{n}(t)$ to denote the amount of actually harvested energy at node $n$, which means we have energy admission control.

Then the energy queue length $E_{n}(t)$ evolves as following

$$
\begin{aligned}
E_{n}(t+1)= & E_{n}(t)-\sum_{\substack{m \in \mathcal{N}_{n}^{(o u t)}\\
}} e_{n m}(t)+\sum_{a \in \mathcal{N}_{n}^{(i n)}} \tilde{e}_{a n}(t) \\
& -P_{n}(t)+h_{n}(t)
\end{aligned}
$$

where $\tilde{e}_{a n}(t)=\beta_{a n} e_{a n}(t)$ denotes the energy arrived at node $n$ from node $a, e_{n m}(t)$ denotes the amount of energy transferred from node $n$ to node $m$, and $0 \leq P_{n}(t) \leq P_{\max }$ denotes the energy used for current data transmission. Here we call $\beta_{n m}$ the efficiency of energy transfer link $[n, m]$ and $0<\beta_{n m}<1$. Note that there is an energy causality constraint, which means at every time, the energy used cannot exceed the energy obtained, i.e.,

$$
P_{n}(t)+\sum_{m \in \mathcal{N}_{n}^{(\text {out })}} e_{n m}(t) \leq E_{n}(t), \forall t, \forall n .
$$

In addition, we assume that there exists $e_{\max }<\infty$ such that $\sum_{m \in \mathcal{N}_{n}^{(\text {out })}} e_{n m}(t) \leq e_{\max }, \forall n, \forall t$ and $h_{\max }<\infty$ such that $\hat{h}_{n}(t) \leq h_{\max }, \forall n, \forall t$.

\section{Utility and Transmission Model}

The utility of our system is defined as a concave and continuing differential function of the average admitted traffic and is denoted by $\sum_{n=1}^{N} U\left(r_{n}\right)$, where $r_{n}=\lim _{t \rightarrow \infty} \frac{1}{T} \sum_{t=1}^{T} A_{n}(t)$.

The objective of our problem is to design an algorithm to maximize the utility while stabilizing the system. The algorithm includes traffic control, power allocation and energy transfer.

\section{QUEUES ANALYSIS}

For the network analysis under the stability constraint, the Lyapunov optimization developed in [13] and [14] is proved to be an efficient way. And in this section, we design the algorithm based on the Lyapunov optimization technique.

\section{A. Energy and Data Aware Algorithm}

Define the Lyapunov Function as

$$
L(t)=\frac{1}{2} \sum_{n=1}^{N} Q_{n}^{2}(t)+\frac{1}{2} \sum_{n=1}^{N}\left[E_{n}(t)-\theta_{n}\right]^{2},
$$

where $\theta_{n}$ is a constant parameter. We denote $Z_{n}(t)=$ $\left\{E_{n}(t), Q_{n}(t)\right\}$ as the system state at node $n$ and $\mathbf{Z}(t)=$ $\left\{Z_{1}(t), Z_{2}(t), \ldots, Z_{n}(t)\right\}$. Then the Lyapunov Drift is defined as

$$
\Delta(t)=\mathbb{E}[L(t+1)-L(t) \mid \mathbf{Z}(t)] .
$$

Proposition. 1: The Lyapunov Drift function (7) is upper bounded by

$$
\begin{aligned}
& \Delta(t) \leq B-\mathbb{E}\left\{\sum_{n=1}^{N}\left[\hat{E}_{n}(t)\right]\left[P_{n}(t)-h_{n}(t)\right] \mid \mathbf{Z}(t)\right\} \\
& -\mathbb{E}\left\{\sum_{n=1}^{N} \sum_{m \in \mathcal{N}_{n}^{(\text {out })}} e_{n m}(t) \beta_{n m}\left[\hat{E}_{n}(t)-\hat{E}_{m}(t)\right] \mid \mathbf{Z}(t)\right\} \\
& -\mathbb{E}\left\{\sum_{n=1}^{N} Q_{n}(t)\left[\mu_{n}(t)-A_{n}(t)\right] \mid \mathbf{Z}(t)\right\}
\end{aligned}
$$

where $\hat{E}_{n}(t)=E_{n}(t)-\theta_{n}, \forall n$.

Proof: The Lyapunov Function has two parts, i.e., the energy queue part and the data queue part. 
For the energy queue part, using Eq. (4) we have

$$
\begin{aligned}
& \frac{1}{2}\left[E_{n}(t+1)-\theta_{n}\right]^{2}-\frac{1}{2}\left[E_{n}(t)-\theta_{n}\right]^{2} \\
& \leq-\left[E_{n}(t)-\theta_{n}\right]\left[P_{n}(t)-h_{n}(t)\right] \\
& -\left[E_{n}(t)-\theta_{n}\right]\left[\sum_{m \in \mathcal{N}_{n}^{(\text {out })}} e_{n m}(t)-\sum_{a \in \mathcal{N}_{n}^{(i n)}} e_{a n}(t)\right]+ \\
& {\left[P_{n}(t)+\sum_{m \in \mathcal{N}_{n}^{\text {(out })}} e_{n m}(t)\right]^{2}+\left[\sum_{a \in \mathcal{N}_{n}^{(i n)}} e_{a n}(t)+h_{n}(t)\right]^{2} .}
\end{aligned}
$$

Using the fact that

$$
\begin{aligned}
& {\left[P_{n}(t)+\sum_{m \in \mathcal{N}_{n}^{(\text {out })}} e_{n m}(t)\right]^{2}+\left[\sum_{a \in \mathcal{N}_{n}^{(\text {in })}} e_{a n}(t)+h_{n}(t)\right]^{2}} \\
& \leq\left[P_{\max }+d_{\max } e_{\max }\right]^{2}+\left[d_{\max } e_{\max }+h_{\max }\right]^{2}
\end{aligned}
$$

and defining $\hat{B}=\left[P_{\max }+d_{\max } e_{\max }\right]^{2}+\left[d_{\max } e_{\max }+h_{\max }\right]^{2}$, we have

$$
\begin{aligned}
& \frac{1}{2}\left[E_{n}(t+1)-\theta_{n}\right]^{2}-\frac{1}{2}\left[E_{n}(t)-\theta_{n}\right]^{2} \\
& \leq \hat{B}-\left[E_{n}(t)-\theta_{n}\right]\left[P_{n}(t)-h_{n}(t)\right] \\
& -\left[E_{n}(t)-\theta_{n}\right]\left[\sum_{m \in \mathcal{N}_{n}^{(\text {out })}} e_{n m}(t)-\sum_{a \in \mathcal{N}_{n}^{(i n)}} e_{a n}(t)\right] .
\end{aligned}
$$

By summing (10) over all $n$ and rearranging the terms, we have

$$
\begin{aligned}
& \frac{1}{2} \sum_{n=1}^{N}\left[E_{n}(t+1)-\theta_{n}\right]^{2}-\frac{1}{2} \sum_{n=1}^{N}\left[E_{n}(t)-\theta_{n}\right]^{2} \\
& \leq N \hat{B}-\sum_{n=1}^{N}\left[E_{n}(t)-\theta_{n}\right]\left[P_{n}(t)-h_{n}(t)\right] \\
& -\sum_{n=1}^{N} e_{n m}(t)\left[\hat{E}_{n}(t)-\beta_{n m} \hat{E}_{m}(t)\right] .
\end{aligned}
$$

Using the fact that $\left(1-\beta_{n m}\right) \hat{E}_{n}(t) \geq-\left(1-\beta_{n m}\right) \theta_{n}$ and defining $\hat{B}^{\prime}=N \hat{B}+\sum_{n=1}^{N} \sum_{m=1}^{N}\left(1-\beta_{n m}\right)$, we have

$$
\begin{aligned}
& \frac{1}{2} \sum_{n=1}^{N}\left[E_{n}(t+1)-\theta_{n}\right]^{2}-\frac{1}{2} \sum_{n=1}^{N}\left[E_{n}(t)-\theta_{n}\right]^{2} \\
& \leq \hat{B}-\sum_{n=1}^{N}\left[\hat{E}_{n}(t)\right]\left[P_{n}(t)-h_{n}(t)\right] \\
& -\sum_{n=1}^{N} e_{n m}(t)\left[\beta_{n m} \hat{E}_{n}(t)-\beta_{n m} \hat{E}_{m}(t)\right] .
\end{aligned}
$$

Using a similar approach, from Eq. (3) we get that

$$
\begin{aligned}
& \frac{1}{2}\left[Q_{n}(t+1)\right]^{2}-\frac{1}{2}\left[Q_{n}(t)\right]^{2} \\
\leq & \hat{B}^{\prime \prime}-Q_{n}(t)\left[\mu_{n}(t)-A_{n}(t)\right],
\end{aligned}
$$

where $\hat{B}^{\prime \prime}=A_{\max }^{2}+\mu_{\max }^{2}$.

Then by summing (13) over all $n$ with (12) and by defining $B=\hat{B}^{\prime}+N \hat{B}^{\prime \prime}$, it comes to our conclusion.
We present the Energy and Data Aware algorithm based on the Lyapunov Drift plus Penalty, which is defined as

$$
\Delta_{V}(t) \triangleq \Delta(t)-V \mathbb{E}\left\{\sum_{n=1}^{N} U\left[A_{n}(t)\right] \mid \mathbf{Z}(t)\right\},
$$

where $V$ is a control parameter.

The idea of the algorithm is to minimize the upper bound of (14) subject to the energy causality constraint (5) as following,

$$
\begin{aligned}
\max : & \sum_{n=1}^{N}\left\{V U\left[A_{n}(t)\right]-Q_{n}(t) A_{n}(t)\right\} \\
& +\sum_{n=1}^{N} \sum_{m \in \mathcal{N}_{n}^{(\text {out })}} e_{n m}(t) \beta_{n m}\left[\hat{E}_{n}(t)-\hat{E}_{m}(t)\right] \\
& +\sum_{n=1}^{N} Q_{n}(t) \mu_{n}(t)+\sum_{n=1}^{N}\left[E_{n}(t)-\theta_{n}\right] P_{n}(t) \\
& +\sum_{n=1}^{N}\left[E_{n}(t)-\theta_{n}\right] h_{n}(t) \\
& P_{n}(t)+\sum_{m \in \mathcal{N}_{n}^{(\text {out })}} e_{n m}(t) \leq E_{n}(t), \forall n .
\end{aligned}
$$

Energy and Data Aware Algorithm: At every time slot $t$, observe $Q_{n}(t), E_{n}(t), \gamma_{n}(t), \forall n$, and do:

- Energy Harvesting: If $E_{n}(t)-\theta_{n}<0$, harvest all the available energy $h_{n}(t)=\hat{h}_{n}(t)$. Otherwise set $h_{n}(t)=0$, which means that there is no need to harvest energy.

- Traffic Admission: Admit $A_{n}(t)$ traffic to the optimal solution of the following problem,

$\max : V U\left(A_{n}(t)\right)-A_{n}(t) Q_{n}(t)$, s.t. $\quad 0 \leq A_{n}(t) \leq A_{\max }$.

- Power Allocation: Define the weight of conveyed energy over link $[n, m]$ at node $n$ as

$$
W_{[n, m]} \triangleq \beta_{n m} \hat{E}_{n}(t)-\beta_{n m} \hat{E}_{m}(t)-\tau .
$$

And define $W_{n}(t)=\max W_{[n, m]}$. Here $\tau=$ $d_{\max } \beta_{\max } e_{\max }+\theta_{\max }, \beta_{\max }{ }^{m}=\max _{n, m} \beta_{n m}$ and $\theta_{\max }=$ $\max \left(\theta_{n}\right)$. Solve the following optimization problem to decide the energy allocation between data transmission and energy transfer,

$$
\begin{aligned}
& \max : \sum_{n=1}^{N} Q_{n}(t) \mu_{n}(t)+\sum_{n=1}^{N} \hat{E}_{n}(t) P_{n}(t) \\
& +\sum_{n=1}^{N} \sum_{m \in \mathcal{N}_{n}^{(\text {out })}} e_{n m}(t)\left[\beta_{n m} \hat{E}_{n}(t)-\beta_{n m} \hat{E}_{m}(t)-\tau\right] \\
& \text { s.t. } P_{n}(t)+\sum_{m \in \mathcal{N}_{n}^{\text {(out })}} e_{n m}(t) \leq E_{n}(t)
\end{aligned}
$$

In the next subsection, we will show this part can be simplified. 


\section{B. Performance Analysis}

In this subsection, we present the performance of the above algorithm. Based on the results, the power allocation in the proposed algorithm can be simplified. The results are given under i.i.d. network randomness. The parameter $\theta_{n}$ is defined as

$$
\theta_{n} \triangleq \delta\left(\alpha_{n} V+A_{\max }\right)+P_{\max }+e_{\max }
$$

where $\alpha_{n}$ is the largest first derivative of the utility function, i.e., $\alpha_{n}=U_{n}^{\prime}(0) \geq U_{n}^{\prime}(t), \forall t$.

Proposition 2: Under the proposed algorithm, we have,

(a) The data queues and the energy queues are bounded, i.e.,

$$
\begin{aligned}
& 0 \leq Q_{n}(t) \leq \alpha_{n} V+A_{\max }, \forall n \\
& 0 \leq E_{n}(t) \leq \theta_{\max }+h_{\max }+d_{\max } \beta_{\max } e_{\max }, \forall n
\end{aligned}
$$

(b) $E_{n}(t) \geq P_{\max }+e_{\max }$ if $P_{n}(t)>0$ or $e_{n m}(t)>0$.

Proof:

Part (a):

For the data queue $Q_{n}(t)$, from (17) we know that if the node $n$ decides to admit new traffic, we must have $Q_{n}(t) \leq$ $V U_{n}^{\prime}(0)=V \alpha_{n}$. So the queue length is always no lager than $\alpha_{n} V+A_{\max }$.

For the energy queue $E_{n}(t)$, it holds for $t=0$ because we assume there is no energy in the buffer at first. Assuming $E_{n}(t) \leq \theta_{n}+h_{\max }+d_{\max } \beta_{\max } e_{\max }, \forall n$ at $t$, we show that it holds for time $t+1$. First if $E_{n}(t) \leq \theta_{n}$, it holds because the maximum input energy is $h_{\max }+d_{\max } \beta_{\max } e_{\max }$. Then if $E_{n}(t) \geq \theta_{n}$, we know it cannot harvest energy but just receive energy from other nodes. If it receives energy from node $m$, then

$$
E_{n}(t)<E_{m}(t)-\theta_{m}-\frac{\tau}{\beta_{n m}}+\theta_{n}<E_{m}(t)+\theta_{n}-\tau
$$

Using the fact that $E_{m}(t) \leq \theta_{\max }+h_{\max }+d_{\max } \beta_{\max } e_{\max }$ and the maximum energy transferred from other nodes is no lager than $d_{\max } \beta_{\max } e_{\max }$, we know that (21) holds for time $t+1$ as well.

Part (b):

Actually we are trying to optimize the following problem,

$$
\begin{aligned}
\max & G\left(e_{n m}(t), P_{n}(t)\right)= \\
& \sum_{n=1}^{N} \sum_{m \in \mathcal{N}_{n}^{(\text {out })}} e_{n m}(t)\left[\beta_{n m} \hat{E}_{n}(t)-\beta_{n m} \hat{E}_{m}(t)-\tau\right] \\
& +\sum_{n=1}^{N} Q_{n}(t) \mu_{n}(t)+\sum_{n=1}^{N} \hat{E}_{n}(t) P_{n}(t) .
\end{aligned}
$$

If $E_{n}(t)<P_{\max }+e_{\max }$, we know that there is no opportunity for the node to transfer energy because $W_{[n, m]}<0$. And if there exits $P_{n}^{*}(t)>0$ to be the optimal solution, we create a new solution to make $P_{n}(t)=0$ and other $P_{m}(t), \forall m \neq n$ and $e_{n m}(t)$ the same. Then,

$$
\begin{aligned}
& G\left(P_{n}^{*}(t)\right)-G\left(P_{n}(t)\right) \\
& =Q_{n}(t) \mu_{n}\left(P_{n}^{*}(t)\right)+\left(E_{n}(t)-\theta_{n}\right) P_{n}^{*}(t) \\
& <\left(\alpha_{n} V+A_{\max }\right) \delta P_{n}^{*}(t)-\delta\left(\alpha_{n} V+A_{\max }\right) P_{n}^{*}(t) \\
& =0
\end{aligned}
$$

This shows that $P_{n}^{*}(t)>0$ cannot be the optimal solution if $E_{n}(t)<P_{\max }+e_{\max }$. Hence it comes to our conclusion and shows that the constraint (5) is indeed redundant.

Based on the Proposition 2, we get the power allocation of the proposed algorithm as following.

- If $E_{n}(t) \leq \theta_{n}$, the node $n$ does not transfer energy to other nodes and does not transmit data either.

- If $E_{n}(t) \geq \theta_{n}$ and $W_{n}(t) \leq 0$, the node $n$ does not transfer energy to other nodes but transmit data using the power of $P_{\max }$.

- If $W_{n}(t)>0$, the node $n$ transfer energy to the node $w$ only with the energy of $e_{\max }$ and transmit data using the power of $P_{\max }$. Here node $w$ satisfies $W_{[n, w]}(t)=$ $W_{n}(t)$.

An important note is that $E_{n}(t) \leq \theta_{n}$ indicates $W_{n}(t) \leq 0$.

Proposition 3: Let the optimal admitted average data rate vector be $\mathbf{r}^{*}$, which is achieved over the class of stationary and randomized policies [10], and let the time average admitted rate vector up to time $T$ achieved by the EDA Algorithm be $\mathbf{r}(T)$. Then, we have

$$
\begin{aligned}
& \liminf _{T \rightarrow \infty} \hat{U}(\mathbf{r}(T))=\liminf _{T \rightarrow \infty}\left\{\sum_{n=1}^{N} U\left[\frac{1}{T} \sum_{t=0}^{T-1} \mathbb{E}\left[A_{n}(t)\right]\right]\right\} \\
& \geq \hat{U}\left(\mathbf{r}^{*}\right)-\frac{\tilde{B}}{V},
\end{aligned}
$$

where $\hat{U}(\mathbf{r}(T))=\sum_{n=1}^{N} U\left(\frac{1}{T} \sum_{t=0}^{T-1} A_{n}(t)\right)$ and $\tilde{B}=B+$ $N d_{\max } e_{\max } \tau$.

Proof: Firstly, we show that the proposed algorithm approximately minimizes the upper bound of (14). Note that the EDA Algorithm indeed maximizes the following function at time $t$ :

$$
\begin{aligned}
D(t)= & \sum_{n=1}^{N}\left\{V U\left[A_{n}(t)\right]-Q_{n}(t) A_{n}(t)\right\} \\
& +\sum_{n=1}^{N} \sum_{m \in \mathcal{N}_{n}^{\text {(out })}} e_{n m}(t) \beta_{n m}\left[\hat{E}_{n}(t)-\hat{E}_{m}(t)-\tau\right] \\
& +\sum_{n=1}^{N} Q_{n}(t) \mu_{n}(t)+\sum_{n=1}^{N}\left[E_{n}(t)-\theta_{n}\right] P_{n}(t) \\
& +\sum_{n=1}^{N}\left[E_{n}(t)-\theta_{n}\right] h_{n}(t)
\end{aligned}
$$

without the energy causality constraint. Next, define $\tilde{D}(t)$ as 
follows:

$$
\begin{aligned}
\tilde{D}(t)= & \sum_{n=1}^{N}\left\{V U\left[A_{n}(t)\right]-Q_{n}(t) A_{n}(t)\right\} \\
& +\sum_{n=1}^{N} \sum_{m \in \mathcal{N}_{n}^{(\text {out })}} e_{n m}(t) \beta_{n m}\left[\hat{E}_{n}(t)-\hat{E}_{m}(t)\right] \\
& +\sum_{n=1}^{N} Q_{n}(t) \mu_{n}(t)+\sum_{n=1}^{N}\left[E_{n}(t)-\theta_{n}\right] P_{n}(t) \\
& +\sum_{n=1}^{N}\left[E_{n}(t)-\theta_{n}\right] h_{n}(t)
\end{aligned}
$$

Note that $\tilde{D}(t)$ is the drift bound of (14) without expectation. The relationship between $\tilde{D}(t)$ and $D(t)$ is shown as:

$$
D(t)=\tilde{D}(t)-\sum_{n=1}^{N} \sum_{m \in \mathcal{N}_{n}^{(\text {out })}} e_{n m}(t) \tau
$$

Since the EDA Algorithm maximizes $D(t)$, we have

$$
\begin{aligned}
& \tilde{D}^{E}(t)-\sum_{n=1}^{N} \sum_{m \in \mathcal{N}_{n}^{(\text {out })}} e_{n m}{ }^{E}(t) \tau \\
& \geq \tilde{D}^{A L T}(t)-\sum_{n=1}^{N} \sum_{m \in \mathcal{N}_{n}^{(\text {out })}} e_{n m}{ }^{A L T}(t) \tau
\end{aligned}
$$

where the superscript $E$ represents the EDA Algorithm, and $A L T$ represents any other alternate policy. Since

$$
0 \leq \sum_{n=1}^{N} \sum_{m \in \mathcal{N}_{n}^{(\text {out })}} e_{n m}(t) \tau \leq N d_{\max } e_{\max } \tau
$$

we have

$$
\tilde{D}^{E}(t) \geq \tilde{D}^{A L T}(t)-N d_{\max } e_{\max } \tau
$$

It means that, the value of $\tilde{D}(t)$ achieved by the proposed algorithm is no less than its value of any other alternative policy minus a constant, including those without the energy causality constraint. In Proposition 2, it has been shown that the energy causality is naturally satisfied under the proposed policy without explicitly being forced. Therefore, (14) can be rewritten as:

$\Delta(t)-V \mathbb{E}\left\{\sum_{n=1}^{N} U\left[A_{n}(t)\right] \mid \mathbf{Z}(t)\right\} \leq B-\mathbb{E}\left\{\tilde{D}^{E}(t) \mid \mathbf{Z}(t)\right\}$.

With (31), we have:

$\Delta(t)-V \mathbb{E}\left\{\sum_{n=1}^{N} U\left[A_{n}(t)\right] \mid \mathbf{Z}(t)\right\} \leq \tilde{B}-\mathbb{E}\left\{\tilde{D}^{A L T}(t) \mid \mathbf{Z}(t)\right\}$,

where $\tilde{B}=B+N d_{\max } e_{\max } \tau$. With the definition of $\tilde{D}(t)$, there always exists an alternative policy satisfying $\tilde{D}^{A L T}(t) \geq$ $V \hat{U}\left(\mathbf{r}^{*}\right)$, where $\mathbf{r}^{*}$ represents the optimal value achieved over the class of stationary randomized policies [10]. Therefore, we have

$$
\Delta(t)-V \mathbb{E}\left\{\sum_{n=1}^{N} U\left[A_{n}(t)\right] \mid \mathbf{Z}(t)\right\} \leq \tilde{B}-V \hat{U}\left(\mathbf{r}^{*}\right)
$$

Taking expectation over $\mathbf{Z}(t)$ and summing (34) over $t \in$ $\{0,1, \ldots, T-1\}$, we have

$\mathbb{E}[L(T)-L(0)]-V \sum_{t=0}^{T-1} \mathbb{E}\left\{\sum_{n=1}^{N} U\left[A_{n}(t)\right]\right\} \leq \tilde{B} T-V \hat{U}\left(\mathbf{r}^{*}\right) T$.

With the non-negative property of Lyapunov functions and $L(0)=0$, we have:

$$
\frac{1}{T} \sum_{t=0}^{T-1} \mathbb{E}\left\{\sum_{n=1}^{N} U\left[A_{n}(t)\right]\right\} \geq \hat{U}\left(\mathbf{r}^{*}\right)-\frac{\tilde{B}}{V}
$$

With the Jensen's inequality and taking a $\lim$ inf as $T \rightarrow \infty$, we have

$$
\liminf _{T \rightarrow \infty}\left\{\sum_{n=1}^{N} U\left[\frac{1}{T} \sum_{t=0}^{T-1} \mathbb{E}\left[A_{n}(t)\right]\right]\right\} \geq \hat{U}\left(\mathbf{r}^{*}\right)-\frac{\tilde{B}}{V}
$$

This completes the proof.

Based on the Proposition 2 and Proposition 3, we have the following observation. Taking $\varepsilon=1 / V$, Part (a) of Proposition 2 shows that the data queue is upper bounded by $O(1 / \varepsilon)$. Combining this result with Proposition 3, we see that the proposed EDA algorithm achieves the utility-backlog tradeoff of $[O(\varepsilon), O(1 / \varepsilon)]$. Furthermore, we also know that the energy queue size is upper bounded by the size of $O(1 / \varepsilon)$. These results provide practical application using our EDA algorithm.

\section{Simulation}

In this section, we present simulation results of the EDA algorithm. The topology of the system is shown in Fig. 1. We assume that the available energy $\hat{h}_{n}(t)$ takes value of $\{70 \mathrm{~J}, 71 \mathrm{~J}, \ldots, 90 \mathrm{~J}\}$ with equal probability for node 2 and node 5 while $\{1 \mathrm{~J}, 2 \mathrm{~J}, \ldots, 10 \mathrm{~J}\}$ with equal probability for node 1 and node 4 . Node 3 can not harvest any energy because of bad weather condition. The energy transfer efficiency is given as $\beta_{1,2}=0.4, \beta_{2,3}=\beta_{3,2}=0.5, \beta_{2,4}=0.3, \beta_{4,5}=\beta_{5,4}=$ 0.8 . We assume $A_{\max }=3 \mathrm{bits}, P_{\max }=10 \mathrm{~J} / \mathrm{slot}, e_{\max }=$ $10 \mathrm{~J} / \mathrm{slot}$ and the utility functions are given as $U_{1}(r)=$ $U_{2}(r)=U_{3}(r)=U_{4}(r)=\log (1+r)$ and $U_{5}(r)=0$. The channel gain $\gamma_{n}(t)$ is assumed to be $\{1,2\}$ with equal probability. In this case, we can use $d_{\max }=2, \alpha_{n}=1(n=$ $1,2,3,4), \alpha_{5}=0, \delta=2$ and $\theta_{n}, \tau$ can be calculate as mentioned before. We simulate $V \in\{1,5,10,20,30,40,50\}$. Each simulation run for $5 \times 10^{5}$ slots. 


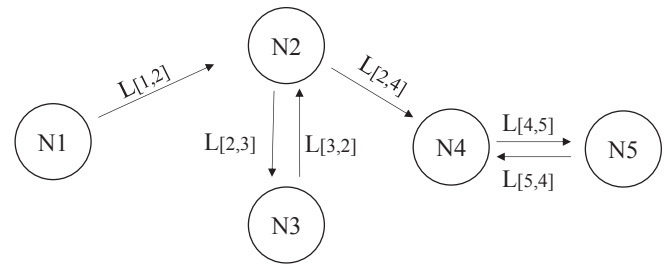

Fig. 1. Topology of the simulation system

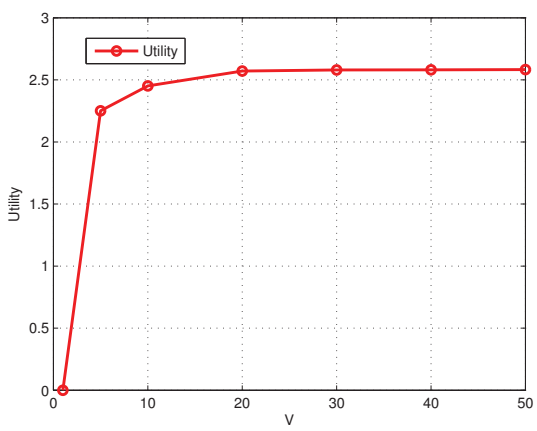

Fig. 2. Utility of the system under different V

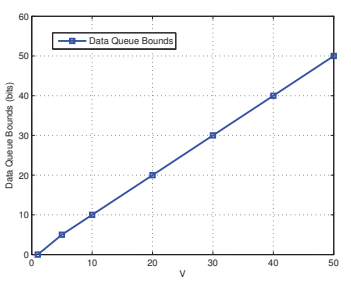

(a) Data Queue Bounds

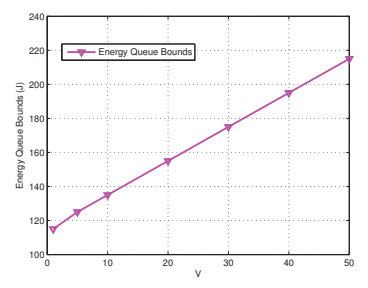

(b) Energy Queue Bounds
Fig. 3. Queue bounds of the system under different V

The results are shown in Fig. 2 and Fig. 3. We can see that the total network utility converges very quickly, which is roughly 2.95 in this example. In addition, data queue bounds and energy queue bounds all grow linearly with $V$, which is the case as we analyzed in previous sections. For better verification of the queueing bounds, we also present the queue processes for node 2 and node 4 under $V=30$ in Fig. 4. It can be seen that the queue sizes are all bounded.

\section{CONCLUSION}

In this paper, we consider the utility optimal problem in energy cooperation networks powered by renewable energy. We propose Energy and Data Aware (EDA) algorithm, which is based on causal information, to achieve an average utility that is within $O(\varepsilon)$ of the optimal for any $\varepsilon>0$ using the energy storage capacity of $O(1 / \varepsilon)$, while keeping the data queue upper bounded by the size of $O(1 / \varepsilon)$.

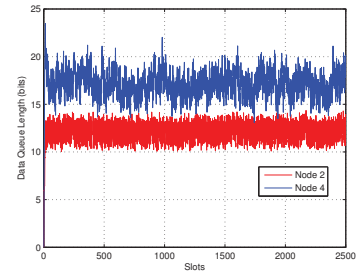

(a) Data Queue Process

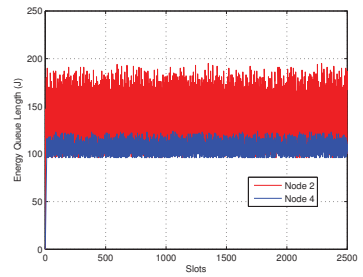

(b) Energy Queue Process
Fig. 4. Queue Processes

\section{ACKNOWLEDGMENT}

We would like to thank Longbo Huang for his insightful advice on the algorithm design.

\section{REFERENCES}

[1] (2010, June) GSMA bi-annnual report: Green power for mobile. [Online]. Available: http://www.gsma.com/developmentfund/wpcontent/uploads/2012/05/GPM Bi-Annual Report June 10.pdf.

[2] M. Gorlatova, A. Wallwater, and G. Zussman, "Networking low-power energy harvesting devices: measurements and algorithms", Proc. of IEEE INFOCOM, Shanghai, pp. 1602-1610, April 2011.

[3] O. Ozel, K. Tutuncuoglu, J. Yang, S. Ulukus, and A. Yener, "Transmission with energy harvesting nodes in fading wireless channels: optimal policies", IEEE J. Selected Area Communications, Vol. 29, No. 8, Sept. 2011.

[4] J. Yang, O. Ozel, and S. Ulukus, "Broadcasting with an energy harvesting rechargeable transmitter", IEEE Trans. on Wireless Communications, Vol. 11, Issue 2, pp. 571-583, February 2012.

[5] J. Yang and S. Ulukus, "Optimal packet scheduling in a multiple access channel with rechargeable nodes", Proc. of IEEE ICC, Kyoto, June 2011.

[6] C. Hu, J. Gong, X. Wang, S. Zhou, and Z. Niu, "Spatial-Temporal WaterFilling Power Allocation in MIMO Systems with Harvested Energy", IEEE ICCC, Xi'an, China, Aug. 2013, accepted.

[7] A. Kansal, J. Hsu, S.Zahedi, and M. B. Srivastava, "Power management in energy harvesting sensor networks", ACM Trans. on Embedded Computing Systems, Vol. 9, No. 2, Feb 2010.

[8] L. Cai, Y. Liu, H. Luan, X. Shen, J.W. Mark, and H. V. Poor, "Dimensioning network deployment and resource management in green mesh networks", IEEE Wireless Communications, vol.18, no.5, pp.58-65, Oct. 2011.

[9] L. Cai Y. Liu, H. Luan, X. Shen, J.W. Mark, and H. V. Poor, "Adaptive resource management in green mesh networks with sustainable energy supply", Proc. of IEEE GLOBECOM, Dec. 2011, pp.1-6.

[10] L. Huang and M. J. Neely, "Utility Optimal Scheduling in Energy Harvesting Networks", IEEE/ACM Trans. on Networking, to appear

[11] M. Zheng, P. Pawelczak, and S. Stanczak, "Planning of Cellular Networks Enhanced by Energy Harvesting", IEEE Communications Letters, 2013, accepted.

[12] Y. K. Chia, S. Sun, and R. Zhang, "Energy Cooperation in Cellular Networks with Renewable Powered Base Stataions", Proc. of IEEE WCNC, April, 2013.

[13] M. J. Neely. "Energy optimal control for time-varying wireless networks", IEEE Trans. on Information Theory 52(7): 2915-2934, July 2006.

[14] L. Huang and M. J. Neely. "Utility optimal scheduling in processing networks", Proc. of IFIP Performance, 2011. 